\title{
Acknowledgment to Reviewers of Axioms in 2021
}

\author{
Axioms Editorial Office
}

Citation: Axioms Editorial Office. Acknowledgment to Reviewers of Axioms in 2021. Axioms 2022, 11, 54. https://doi.org/10.3390/axioms11020054

Published: 28 January 2022

Publisher's Note: MDPI stays neutral with regard to jurisdictional claims in published maps and institutional affiliations.

Copyright: (C) 2022 by the author. Licensee MDPI, Basel, Switzerland. This article is an open access article distributed under the terms and conditions of the Creative Commons Attribution (CC BY) license (https://creativecommons.org/licenses/by/4.0/).

MDPI AG, St. Alban-Anlage 66, 4052 Basel, Switzerland

Rigorous peer-reviews are the basis of high-quality academic publishing. Due to the great efforts of our reviewers, Axioms was able to maintain its standards for the high quality of its published papers. Thanks to the contribution of our reviewers, in 2021, the median time to first decision was 22 days, and the median time to publication was 43 days. The editors would like to extend their gratitude and recognition to the following reviewers for their precious time and dedication, regardless of whether the papers they reviewed were finally published:

A. K. Lazopoulos

Adam Lecko

Adam Pacławski

Adel Nailevich Abyzov

Adrian Gligor

Alef Sterk

Aleksandr Murach

Aleksandr Rakhmangulov

Aleksandra Tutueva

Alessandro Oliaro

Alexander V. Mantzaris

Alexei Sergeevich Shvedov

Alexey S. Eremin

Alim Sukhtayev

Alina-Mihaela Patriciu

Aliona Dreglea

Allison Lewis

Amaury Hayat

Ana Vukelić

Anatolij Prykarpatski

Andrea Scapellato

Andreea Fulga

Andrei Horvat-Marc

Andrés Carrión

Andrey Cherstvy

Andrey Morgulis

Andrey Zahariev

Andriy Bandura

Andriy Zagorodnyuk

Andrzej Bielecki

Apostolos Syropoulos

Arianna D'Ulizia

Arkadiusz Kawa

Arni S.R. Srinivasa Rao
Arran Fernandez

Arsen Palestini

Arseny Shur

Arthur E. P. Veldman

Artur Kobus

Atanaska Georgieva

Aviv Gibali

Azhar Zafar

Bakhtiyor Jalilovich Kadirkulov

Balazs Toth

Baltazar Aguirre-Hernández

Benoit Morel

Bernard Linsky

Branislav Randjelovic

Brett Altschul

Brigita Ferčec

Calogero Vetro

Can Baskent

Chih-Wen Chang

Ching-Lueh Chang

Choonkil Park

Christophe Chesneau

Christopher Boyd

Christos Katris

Christos Makris

Constantin Udriste

Cristian Busu

Cristiana De Filippis

Dan Tiba

Dana Smetanová

Dana-Mihaela Petroșanu

Dan-Cristian Dabija

Danial Faghihi

Daniela Marian 
Danijela Tuljak-Suban

Daniyal Haider

Darja Rupnik Poklukar

Darjan Karabasevic

David Orellana Martín

Davor Dragicevic

Dmitriy Kolyukhin

Dorinela Costescu

Dragan Pamucar

Dumitru Baleanu

Dumitru Vieru

Dunhui Xiao

Dusan Rakic

Edward Cartwright

Elio Padoano

Elisabeth Longatte-Lacazedieu

Emanuele Rodaro

Enrique G. Reyes

Erick Méndez

Ernesto Zambrano-Serrano

Ervin K. Lenzi

Eugen Divjak

Ewa Chodakowska

Fabien Crauste

Fabrizio Maturo

Farooq Ahmad

Farooq Gujar

Farrukh Maksutovich Mukhamedov

Ferdinando Di Martino

Fernando Veiga-Suárez

Francesca Pitolli

Francesco Pediconi

Francisco Gallego Lupiaňez

Francisco Javier Garcia-Pacheco

Francisco Jurado

Francisco Martínez González

Francisco Ureña

François Leyvraz

František Klimenda

Fumiya Okubo

Gabriella Puppo

Galina Ilieva

Geneviève Simonet

Georgi Georgiev

Giacomo Dimarco

Gilberto Gonzalez-Parra

Giordano Santilli

Giovanni Angiulli

Giovanni Calvaruso

Goran Cirovic

Gulasekaran Rajaguru
Guoqiang Zeng

Gustavo Fernández-Muñoz

Gustavo Santos-García

Gyurhan Nedzhibov

Hamza Khan

Hao Xiong

Haret Rosu

Hassen Aydi

Helbert Eduardo Espitia

Heyrim Cho

Hijaz Ahmad

Ho Namgung

Houssine Zine

Hovik Matevossian

Hui-Chin Tang

Huseyin Isik

Hussein Rappel

Hyunwook Koh

Igor Andrianov

Igor Vujović

Ilya Boykov

Ilya G. Kovalenko

Indra Gunawan

Ioannis Dassios

Ivano Colombaro

Izhar Ahmad

J. Leonel Rocha

J. Vanterler Da C. Sousa

Jakub Horák

Jan Cieslinski

Jan Felipe Van Diejen

Jan Mandel

Jan Van Casteren

Janusz Brzdek

Janusz Matkowski

Javier Fernandez

Jean-Paul Doignon

Jelena Djokić

Jen-Chih Yao

Jen-yao Lee

Jesus Enrique García

Ji-huan He

Jinrong Wang

Jin-Woo Park

Joanna Raczek

João Cabral

Joe Repka

Jong-Chan Kim

Jorge Sanchez-Ortiz

José Alberto Rodrigues

José Antonio de la O. Serna 
José Antonio Oller

José Carlos Alcantud

Jose Martin Castro Manzano

José Matos

Josef Mikeš

Josef Slapal

Juan Carlos Hernández-Gómez

Juan Francisco Sánchez-Pérez

JuingShian Chiou

Junesang Choi

Jun-Lin Lin

Kanel Alexey

Kao-Yi Shen

Karl H. Hofmann

Karl-Joachim Wirths

Katarzyna Antosz

Ketson Roberto Maximiano Dos Santos

Kifayat Ullah

Kirkby Justin Lars

Ki-sun Lee

Konstantin Alexandrovich Rybakov

Konstantin Kudryavtsev

Konstantin Zhukovsky

Kottakkaran Sooppy Nisar

Krassimira Vlachkova

Kudret Topyan

Kuize Zhang

Kyriakos Flouris

Lanre Akinyemi

Laszlo Pokoradi

Lech Górniewicz

Leila Marek-Crnjac

Lenka Mihoković

Lenny Neyt

Lev B. Klebanov

Ljubiša Kočinac

Lloyd Davis

Lorentz Jäntschi

Lorenzo Zanelli

Lotfi Chaari

Lovyagin Yurii

Luca Gemignani

Luis Enrique Valdez-Juárez

Luisa D'Amore

Łukasz Nakonieczny

M. Vicenta Ferrer

Mahmoud Ibrahim

Maksim Kukushkin

Manuel Alberto Martins Ferreira

Manuel De La Sen

Marcelina Mocanu
Marcello Delitala

Marcelo Kaminski Lenzi

Marco Jose

Marek Golasinski

Marek Hycko

Marek Lampart

Marek Sawerwain

Maria Alessandra Ragusa

Maria Dobrițoiu

Maria Korovina

Maria L. Manca

Maria Laura Delle Monache

Marina Resta

Marinela Inta

Marinko Maslaric

Marius Pislaru

Mariusz Buciakowski

Mariusz Pleszczyński

Mariusz Urbański

Marko Kostić

Massimo Tessarotto

Matthew Harrison-Trainor

Matthew J. Lake

Max Bannach

Mehdi Salimi

Mehmet Yavuz

Merlin Simo-Tagne

Michael (Michail) Th. Rassia

Michael Evans

Michel Mehrenberger

Michel Nguiffo Boyom

Mieczyslaw Cichon

Mikhail Babenko

Mikhail Tkachenko

Mimica R. Milošević

Ming Li

Miomir Stankovic

Mirjana Pavlović

Miroslav Hristov

Miroslava Antić

Mohamed Hamdaoui

Monika Kosacka-Olejnik

Mostafa M. A. Khater

Muhammad Fazal Ijaz

Muhammed Mursaleen

Mustafa Avci

Nalini Chintalapudi

Nanjing Hao

Nataliia Melnykova

Naveed Ahmed Azam

Nhon Nguyen-Thanh 
Nikita Y. Lovyagin

Niklas Kolbe

Nikola Lopac

Octav Olteanu

Oleg Gutik

Oleh Romaniv

Olga Chichigina

OPhir Nave

Orlov Viktor Nikolaevich

Oscar Castillo

Oscar Valero

Oswaldo González-Gaxiola

Paolo Emilio Ricci

Paolo Leonetti

Paolo Mason

Paolo Mercorelli

Paolo Musolino

Papadimitriou Fivos

Patricio Ordaz

Paul Popescu

Pavlina Jordanova

Paweł Drąg

Pedro A. Martin Cervantes

Pedro Macedo

Petr Sváček

Piotr Nowak

Pyke Tin

Radu Paltanea

Raimundas Vidunas

Rajkumar Verma

Renat T. Sibatov

Richard Kerner

Roberto Garrappa

Roberto Saia

Robin Hankin

Roman Ivanovich Parovik

Roman M. Cherniha

Ronghua Zhang

Saeid Jafari

Sai Sidhardh

Salissou Moutari

Salvatore Sessa

Samad Noeiaghdam

Sansit Patnaik

Satoshi Uchida

Senol Piskin

Serena Doria

Sergei Aseev

Sergei Pilyugin

Sergey Grigor'evich Pyatkov

Sergey Shmarev
Sergey V. Sudoplatov

Sergii Kavun

Sergii Myroshnychenko

Shahram Rezapour

Silviu Urziceanu

Slobodan Radojevic

Snezhana Hristova

Sorin Dragomir

Stefan Jovčić

Stefan Tappe

Stefka Fidanova

Stepan A. Tersian

Stephen G. Penny

Stevan Pilipović

Stupňanová Andrea

Sun Young Cho

Susmit Bagchi

Svajone Bekesiene

Svetlozar T. Rachev

Svetozar Margenov

Tatjana Dosenovic

Tatjana Petek

Teodor Atanackovic

Teodor Turcanu

Teresa Diogo

Thanin Sitthiwirattham

Theodore Andronikos

Theofanis Grammenos

Thierry Gobron

Thomas Ernst

Thomas Michelitsch

Thyago Nepomuceno

Tibor K. Pogány

Tom Mestdag

Tomas Baležentis

Tomasz Steifer

Tommaso Gentile

Tristan Guillaume

Umar Iqbal

Vasiliev Borisovich Vladimir

Viorel Paleu

Vittorio Astarita

Vladimir E. Fedorov

Vladimir Nikolayevich Soloviev

Vladimir Vasilyev

Wai Siong Chai

Wayne Lewis

Wei Lu

Wei-Lun Tsai

Wei-Shih Du

Wieslaw A. Dudek 
Wiesław Leonski

Wojciech Froelich

Wojciech Sałabun

Xuefeng Zhang

Yagub Sharifov

Yan Pei

Yanqiao Wei

Yingjie Liang

Yoritaka Iwata

Yosuke Kuratomi
Youngchul Bae

Yuri Kordyukov

Yuriy Povstenko

Yury K. Mashunin

Zbigniew Haba

Zhan-Peng Yang

Zhen Zhang

Zhiqiang Wang

Zoran Vosika 